\title{
rESCMA: A brief summary on effect size conversion for meta-analysis
}

\author{
César Villacura-Herrera1 1 (iD \& Nicolas Kenner ${ }^{2}$
}

\begin{abstract}
${ }^{1}$ Laboratory of Methodology for Behavioral Sciences and Neurosciences, Faculty of Psychology, Universidad de Talca, Chile. 2 Goddard School of Business \& Economics, Weber State University, USA.
\end{abstract}

\begin{abstract}
Effect sizes are highly relevant in quantitative research. It facilitates the comparison and quantitative synthesis of scientific studies. The main objective of this report is to present: a) a brief summary of the formulas used for conversion between the three main effect sizes used in the meta-analysis: the correlation coefficient, the standardized mean difference and the odds ratio; and b) the Rapid Effect Size Converter for Meta-Analysis (rESCMA), a open-source and browser-based app for efficient conversion and bulk-conversion of effect sizes and their variances based on the formulas proposed in this report. In addition, a table summarizing the formulas is presented for easy accessibility and use.
\end{abstract}

Keywords: Meta-analysis, effect size, conversion, open-source, rESCMA

\section{Introduction}

In inference statistics, a $p$ value is used as a reference for hypotheses testing. When the value is 0.05 or less $(p<.05)$, it is customary to report that there has been a 'significant' result, whether two or more means are being compared or the strength of the association is being assessed. A main issue with $p$ values, which has previously been addressed by researchers (Goodman, 2008; Lin et al., 2009; Pandis, 2013; Royall, 1986) is that it relies too much on the sample data at hand, which is treated as a population. As a result, the larger the number of samples or number of subjects, the more statistically significant the result will tend to be. This means that while the results for an hypothesis test may not have been particularly significant for a sample of 50 people, it is rather possible that it will for 500 people.

An effect size is an index that shows the strength of an interaction (Field \& Hole, 2003). As mentioned above, a $p$ value changes based on the sample size, so it has a substantial effect. This is to say, it doesn't give by itself any information about whether the interaction is big or small. On the other hand, a huge advantage of most effect sizes is their ability to express the strength of difference, association or interaction without stopping limitations such as sample size or significance tests, also managing to measure a specific parameter in a specified population (Schäfer \& Swartz, 2019).

Overall, standardized effects sizes such as the Correlation Coefficient $(r)$ or the Standardized Mean Difference $(d)$ are preferred when different measurement scales are being used between studies, while unstandardized effect sizes such as the Odds Ratio (OR) and Relative Risk (RR) are usually reported in studies with binary outcomes (Aerts et al., 2013).

Cohen (1988) argued that the size of the effect was formulated as an essential accompaniment to the hypothesis testing, allowing for a straightforward understanding of the magnitude of the phenomena under study, as well as providing a more suitable interpretation of the results and their implications. In addition, effect sizes are a critical component for the integration of different results through meta-analysis (Hunter \& Schmidt, 2004).

In this setting, studies often report enough data to estimate a determined effect size, while others provide enough data to estimate another. If the researcher judges that the two studies address the same subject, we would expect to include both in a quantitative synthesis of the data. In this case, one or both values must be converted to a common standardized effect size measure. In this regard, Borenstein et al. (2009) stated that this only makes complete sense if the studies are comparable in a relevant way, where the fact that different measures were used for effect size estimation will not be an obstacle to the assessment of the study results.

Consequently, it must be acknowledged that all the effect sizes must be characterized within the specific context and field of research, the level of conceptual development and practical application in the world. It can be intricate to make a comparison of the effects between the research findings due to the number of anticipated and unpredictable factors and their possible arrangements.

\section{Converting effect sizes}

The following formulas are mainly based on those proposed by Borenstein et al. (2009) and Botella \& Sánchez (2015). For accuracy, all decimals are shown in the results below.

\subsection{Converting Correlation Coefficients ( $r$ )}

\subsubsection{Odds Ratio (OR)}

If we have the correlation coefficient as a base, we can carry out the transformation to both $O R$ and $d$. Although the value of OR cannot be obtained directly, we can calculate it through its natural logarithmic expression $(\mathrm{LnOR})$ in the following way:

$$
\operatorname{LnOR}=\frac{2 r \cdot \varphi}{\sqrt{1-r^{2}}}
$$

where $\varphi$ is a constant of value 1.81379936423434. For this exercise, we will assume that the value of $r$ is .211. Then, we can determine that: 


$$
\begin{gathered}
\operatorname{LnOR}=\frac{(2(.211)) \cdot 1.8137993642343}{\sqrt{1-0.044521}} \\
\operatorname{LnOR}=\frac{0.765423331706891}{0.977486061281694}=0.78305293755622
\end{gathered}
$$

and in this way, obtain the value of OR through its exponential function:

$$
O R=e^{L n O R}
$$

so that:

$$
\begin{gathered}
O R=e^{0.783052937556222} \\
O R=2.1881423405861
\end{gathered}
$$

\subsubsection{Standardized mean difference (d)}

Once we know the value of $\mathrm{LnOR}$, it is possible to calculate the value of $d$ using the following formula:

$$
d=\operatorname{LnOR} \cdot \vartheta
$$

where $\vartheta$ is a constant of value 0.551328895421756 . With this, we can determine that:

$$
\begin{gathered}
d=(2.1881423405861 \cdot 0.551328895421756) \\
d=0.431719711119633
\end{gathered}
$$

\subsection{Converting Odds Ratio $(O R)$}

In other cases, is it possible that we might only have value of OR and require its conversion to either $r$ or $d$ in order to keep consistency with our main effect size measure. As mentioned above, $O R$ cannot be directly converted into $r$ nor $d$, so it is required to transform it to its natural logarithmic expression:

$$
\operatorname{LnOR}=\log _{e}(O R)
$$

Continuing with the example, let us suppose that we only have the OR value, which is 2.1881423405861 :

$$
\begin{gathered}
\operatorname{LnOR}=\log _{e}(2.1881423405861) \\
\text { LnOR }=0.78305293755622
\end{gathered}
$$

\subsubsection{Correlation coefficient ( $r$ )}

Once the value of $L n O R$ has been determined, the value of $\mathrm{r}$ can be easily calculated using the following formula:

$$
r=\frac{\operatorname{LnOR} \cdot \vartheta}{\sqrt{4+(\operatorname{LnOR} \cdot \vartheta)^{2}}}
$$

so that:

$$
\begin{gathered}
r=\frac{0.78305293755622 \cdot 0.551328895421756}{\sqrt{4+(0.78305293755622 \cdot 0.551328895421756)^{2}}} \\
r=\frac{0.431719711119632}{2.04606498160963}=.211
\end{gathered}
$$

2.2.2. Standardized mean difference $(d)$

Once we have determined the value of both $r$ and $L n O R$, the value of $d$ can be calculated using [2.3].

\subsection{Converting Standardized Mean Differences (d)}

2.3.1. Correlation coefficient $(r)$

In a third scenario, it is possible that we might only have the value of $d$ and require it conversion to either $r$ or $O R$ in order to keep consistency with our main effect size measure. We can convert $d$ into $r$ using the following formula:

$$
r=\frac{d}{\sqrt{4+d^{2}}}
$$

Following the example, let us suppose that we only know the value of $d$, which is 0.431719711119633 . Then:

$$
\begin{aligned}
r & =\frac{0.431719711119633}{\sqrt{4+(0.431719711119633)^{2}}} \\
r & =\frac{0.431719711119633}{2.04606498160963}=.211
\end{aligned}
$$

\subsubsection{Odds Ratio (OR)}

Once we have determined the value of both $d$ and $r$, the value of LnOR can be calculated using [a], and then converted into OR using [b].

\section{Converting sampling variances}

Along with the effect size, the meta-analysis requires knowing their sampling variance. The easiest way to calculate the variance for all three effect sizes is starting with calculating the variance of the correlation coefficient. To do this, the following formula is used:

$$
V_{r}=\frac{\left(1-r^{2}\right)^{2}}{n-1}
$$

where $n$ represents the sample size of the study. In the example where we know that value of $r$ is .211 , we will now assume a sample size of 150 participants. Then:

$$
\begin{gathered}
V_{r}=\frac{\left(1-(.211)^{2}\right)^{2}}{150-1} \\
V_{r}=\frac{(1-0.044521)^{2}}{150-1}=0.00612711489557718
\end{gathered}
$$

From here, the value of $V_{r}$ can be easily converted to obtain the variance of $d$. To do this, we will use the following formula:

$$
V_{d}=\frac{4 \cdot V_{r}}{\left(1-r^{2}\right)^{3}}
$$

so that:

$$
\begin{gathered}
V_{d}=\frac{4 * 0.00612711489557718}{\left(1-(.211)^{2}\right)^{3}} \\
V_{d}=\frac{.0245084595823087}{.872295112383367}=.0280965228789
\end{gathered}
$$

Once $V_{d}$ has been calculated, we can calculate the variance of $\mathrm{LnOR}$ with the following formula:

$$
V_{L n O R}=V_{d} \cdot \varpi
$$


where $\varpi$ is a constant of value 3.28986813369689 , so that:

$$
\begin{gathered}
V_{\text {LnOR }}=.028096522878988 \cdot 3.28986813369689 \\
V_{\text {LnOR }}=0.0924338552872682
\end{gathered}
$$

In $O R$-based meta-analysis, researchers work with $V_{L n O R}$, so no further conversion is required.

\section{Rapid Effect Size Converter for Meta-Analysis (rESCMA)}

Although there are a few effect size converters available online, they lack two essential features for their active use in metaanalytic research: First, the possibility of calculating and converting among sampling variances in a standardized way; second, the ability to bulk-convert effect sizes and directly transfer them to datasets for analysis. To address this issue, we have developed rESCMA as an open-source and browser-based app to convert among the three effect size measures using the formulas presented in this document and summarized in Table 1. rESCMA is available online and can be accessed through the URL rESCMA.com.

\section{Acknowledgements}

This work was supported by the Research Center on Cognitive Sciences (CICC) and the Associative Research Program (PIA) on Cognitive Sciences of the Faculty of Psychology, Universidad de Talca, Chile.

\section{References}

1. Aarts, S., van den Akker, M., \& Winkens, B. (2013). The importance of effect sizes. European Journal of General Practice, 20(1). 61-64. doi.org/10.3109/13814788.2013.818655
2. Borenstein, M., Hedges, L., Higgins, J. \& Rothstein, H. (2009). Introduction to meta-analysis. Wiley. ISBN: 978-0470-05724-7.

3. Botella, J. \& Sánchez, J. (2015). Meta-análisis en ciencias sociales y de la salud. Síntesis. ISBN: 9788490771242.

4. Cohen, J. (1988). Statistical power analysis for the behavioral sciences. Hillsdate, NJ.

5. Field, A.P. \& Hole, G. (2003). How do design and report experiments. London: Sage.

6. Goodman, S. (2008). A dirty dozen: Twelve p-value misconceptions. Seminars in Hematology, 45. doi.org/10.1053/j.seminhematol.2008.04.003.

7. Hunter, J.E. \& Schmidt, F.L. (2004). Methods of metaanalysis: Correcting error and bias in research findings. Thousand Oaks, CA: Sage.

8. Lin, M., Lucas, H.C. \& Shmueli, G. (2009). Too big to fail: Larger samples and false discoveries. doi.org/10.2139/ssrn.1336700.

9. Pandis, N. (2013). The $\mathrm{p}$ value problem. Am J Orthod Dentofacial Orthop. doi.org/10.1016/j.ajodo.2012.10.005.

10. Royall, R.M. (1986). The effect of sample size on the meaning of significance tests. The American Statician, 40(4). doi.org/10.2307/2684616.

11. Schäfer, T. \& Schartz, M.A. (2019). The meaningfulness of effect sizes in psychological research: Differences between sub-disciplines and the impact of potential biases. Frontiers in Psychology, 10. doi.org/10.3389/fpsyg.2019.00813

Table 1

Summary

\begin{tabular}{ccc}
\hline$r$ to $\operatorname{LnOR}$ & $\operatorname{LnOR}$ to $O R$ & $\operatorname{LnOR}$ to $d$ \\
\hline $\operatorname{LnOR}=\frac{2 r \cdot \varphi}{\sqrt{1-r^{2}}}$ & OR $=e^{\operatorname{LnOR}}$ & $d=\operatorname{LnOR} \cdot \vartheta$
\end{tabular}

Converting

effect sizes

\begin{tabular}{|c|c|c|c|}
\hline & $V_{r}$ & $V_{r}$ to $V_{d}$ & $V_{d}$ to $V_{L n O R}$ \\
\hline $\begin{array}{l}\text { Converting } \\
\text { variances }\end{array}$ & $V_{r}=\frac{\left(1-r^{2}\right)^{2}}{n-1}$ & $V_{d}=\frac{4 \cdot V_{r}}{\left(1-r^{2}\right)^{3}}$ & $V_{L n O R}=V_{d} \cdot \varpi$ \\
\hline
\end{tabular}

\begin{tabular}{ccc}
\hline OR to $\operatorname{LnOR}$ & $\operatorname{LnOR\text {to}r}$ & $d$ to $r$ \\
\hline $\operatorname{LnOR}=\log _{e}(O R)$ & $r=\frac{\operatorname{LnOR} \cdot \vartheta}{\sqrt{4+(\operatorname{LnOR} \cdot \vartheta)^{2}}}$ & $r=\frac{d}{\sqrt{4+d^{2}}}$
\end{tabular}

\begin{tabular}{|c|c|c|}
\hline$r$ & $=$ & Correlation coefficient \\
\hline$d$ & $=$ & Standardized mean difference \\
\hline$O R$ & $=$ & Odds Ratio \\
\hline $\operatorname{LnOR}$ & $=$ & Natural logarithm of the Odds Ratio \\
\hline$\varphi$ & $=$ & 1.81379936423434 \\
\hline$\vartheta$ & $=$ & 0.551328895421756 \\
\hline$\varpi$ & $=$ & 3.28986813369689 \\
\hline
\end{tabular}

